N. E. Piskunov, W. W. Weiss, D. F. Gray, eds.

\title{
Round Table Summary: \\ Instrumentation and Data Processing
}

\author{
Saul J. Adelman
}

Department of Physics, The Citadel, 171 Moultrie Street, Charleston, SC 29409, United States of America

\author{
Ilfan Bikmaev \\ Department of Astronomy, Kazan State University, Kremlevskaya Str., \\ 18, Kazan, 420008, Russia \\ Austin F. Gulliver \\ Department of Physics and Astronomy, Brandon University, Brandon, \\ $M B$ R7A 6A9, Canada
}

\author{
Barry Smalley \\ Astrophysics Group, School of Chemistry $\mathcal{G}$ Physics, Keele University, \\ Keele, Staffordshire ST5 5BG, United Kingdom
}

\begin{abstract}
During this round table Bikmaev described the systematic differences among equivalent width measurements and Gulliver discussed the reduction of high dispersion, high signal-to-noise CCD spectra. Smalley considered atmospheric extinction as it affects astronomical measurements while Adelman presented an overview of the ASTRA spectrophotometer.
\end{abstract}

\section{Introduction}

High quantum efficiency array detectors such as CCDs have changed high dispersion spectroscopy in the last few decades. While with photographic plates astronomers achieved signal-to-noise $(\mathrm{S} / \mathrm{N})$ ratios of order 25 , today $\mathrm{S} / \mathrm{N}$ values of 200 are common place with values up to 4000 being achieved. Further astronomers should now be able to better calibrate their data. Some problems such as scattered light in the spectrograph which were previously ignored now need to be considered as their effects can be seen in the data.

Although one might have expected spectroscopists after reducing data of the same star with similar resolutions from different high dispersion spectrographs to obtain essentially identical results, there are still differences especially between echelle and coudé spectrographs. This can be due to several effects including how the scattered light was removed, instrumental profile changes along an echelle order, how the spectra were normalized, and the type of profile used to measure the equivalent widths. All of these effects need to be understood so that any systematic errors can be removed. 
We live at the bottom of an ocean of air. Sunlight and starlight passing through our atmosphere is subject to a general extinction and in addition the imposition of telluric lines and bands. The removal of telluric features from high dispersion spectra is still somewhat of an art. Photometrists and spectrophotometrists have to correct their observations for these effects.

Since the retirement of most of the rotating grating scanners well over a decade ago, stellar astronomy has not had an instrument which could produce high quality spectrophotometry. Now Adelman and his colleagues (Adelman et al. 2002, this conference) are building a multiplexed spectrophotometer with a $\mathrm{CCD}$ as a detector and a $0.5-\mathrm{m}$ automated telescope on which it will be used.

\section{On Systematic Differences in Measured Equvalent Widths of 'Clas- sical' and Echelle Spectrographs}

My colleagues A. Galeev (Kazan State University), F. Musaev, and G. Galazutdinov (Special Astrophysical Observatory of the Russian Academy of Sciences), and I (I. Bikmaev) have compared published equivalent widths (EW) values with our original measures in spectra of the same stars as observed using modern high-resolution echelle spectrographs (including that at the 2-m telescope at Terskol Observatory, Musaev et al. 1999) and classical ones (one-dimensional spectra obtained by long focus cameras equipped with Reticons as a detectors).

Figure 1 demonstrates that echelle systems show very good systematic agreement at the level of 1-2 \%. The reduction of the echelle spectra were based on different packages (IRAF, MIDAS, or our home package DECH for the PC, Galazutdinov 1992)

But there are systematic differences in EWs at the level of 5-15\% between the echelle and 'classical' systems using Reticon detectors. The 'classical' EWs are smaller than those obtained from echelle spectra. These results are independent of spectral type. Figure 2 shows the results for the hot B stars while Figure 3 shows those for the cool F and G stars. Kindly note that the results for the same F-G stars are shown in the Figures 1a,b, 2a,b, and 2c,d.

Improper correction for a spectrograph's scattered light could be one reason for this effect (by increasing the 'continuum level'). The 'background subtraction' option is always used during echelle spectra reductions due to the high level of scattering light produced by the echelle grating. But this option was usually absent for reduction procedures used for one-dimension classical spectra, or the effect of scattered light was not fully removed. Most modern observations are carrying out with echelle spectrographs but much of the archival data were obtained with classical spectrographs and some observatories continue to use these instruments. Systematic differences in EW may lead to systematic (and accidental) errors in the determination of stellar atmospheric parameters $\left(\mathrm{V}_{\text {turb }}, \mathrm{T}_{\text {eff }}, \log \mathrm{g}\right)$ which propagate into the corresponding abundance determinations. As noted below CCD-based observations now do account properly for scattered light; the EWs from these observations should be compared with the early Reticon-based results. If scattered light can be isolated as the cause of the above differences, we suggest that the EWs of a sample of 'standard' stars be obtained using modern echelle spectrographs for use in transforming EWs from 'classical' spectrographs to a homogeneous system (a method which has been 

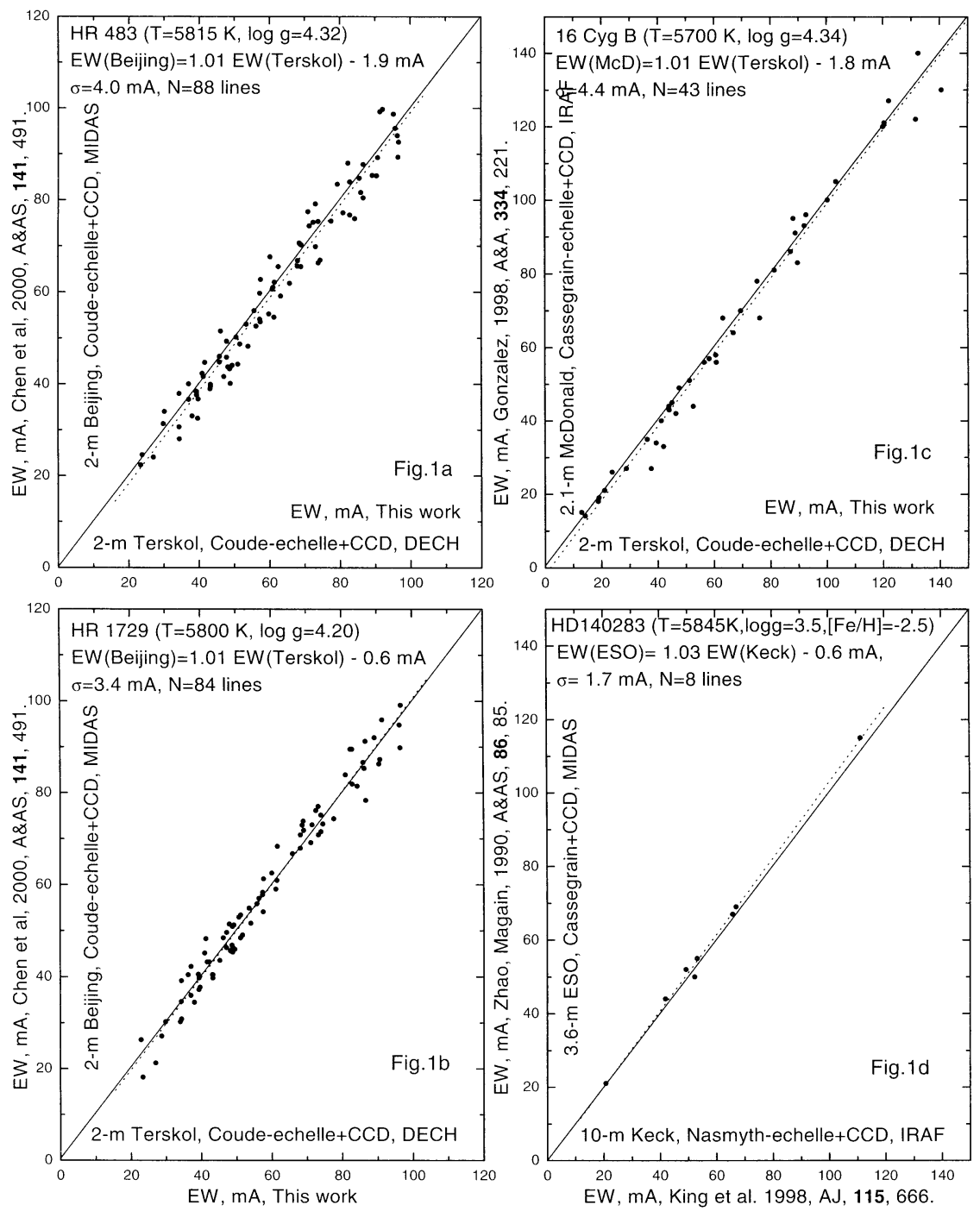

Figure 1. Comparison of EWs measured using different echelle spectrographs and different software 

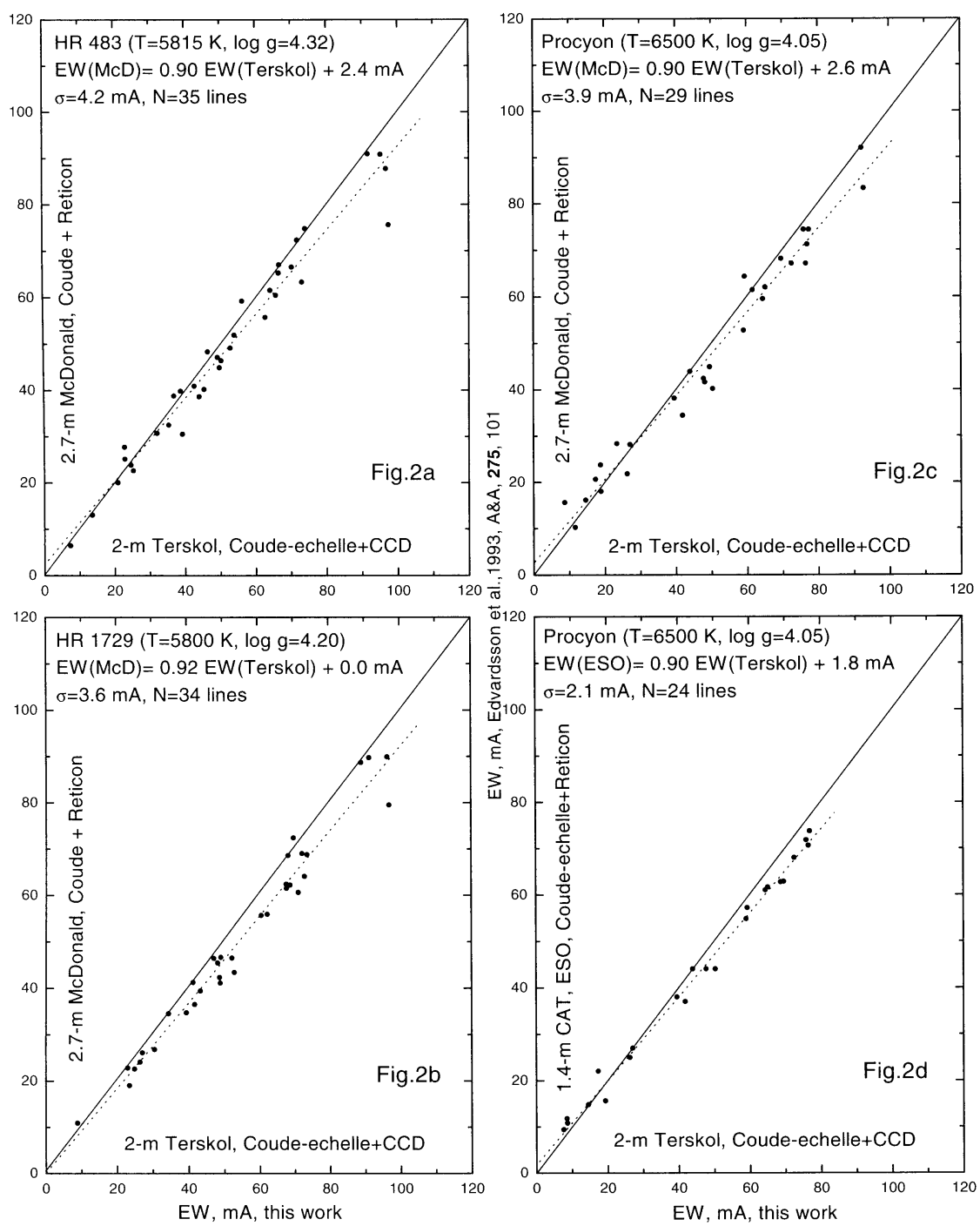

Figure 2. Comparison of EWs measured from 'classical' and echelle spectrographs for F-G stars 

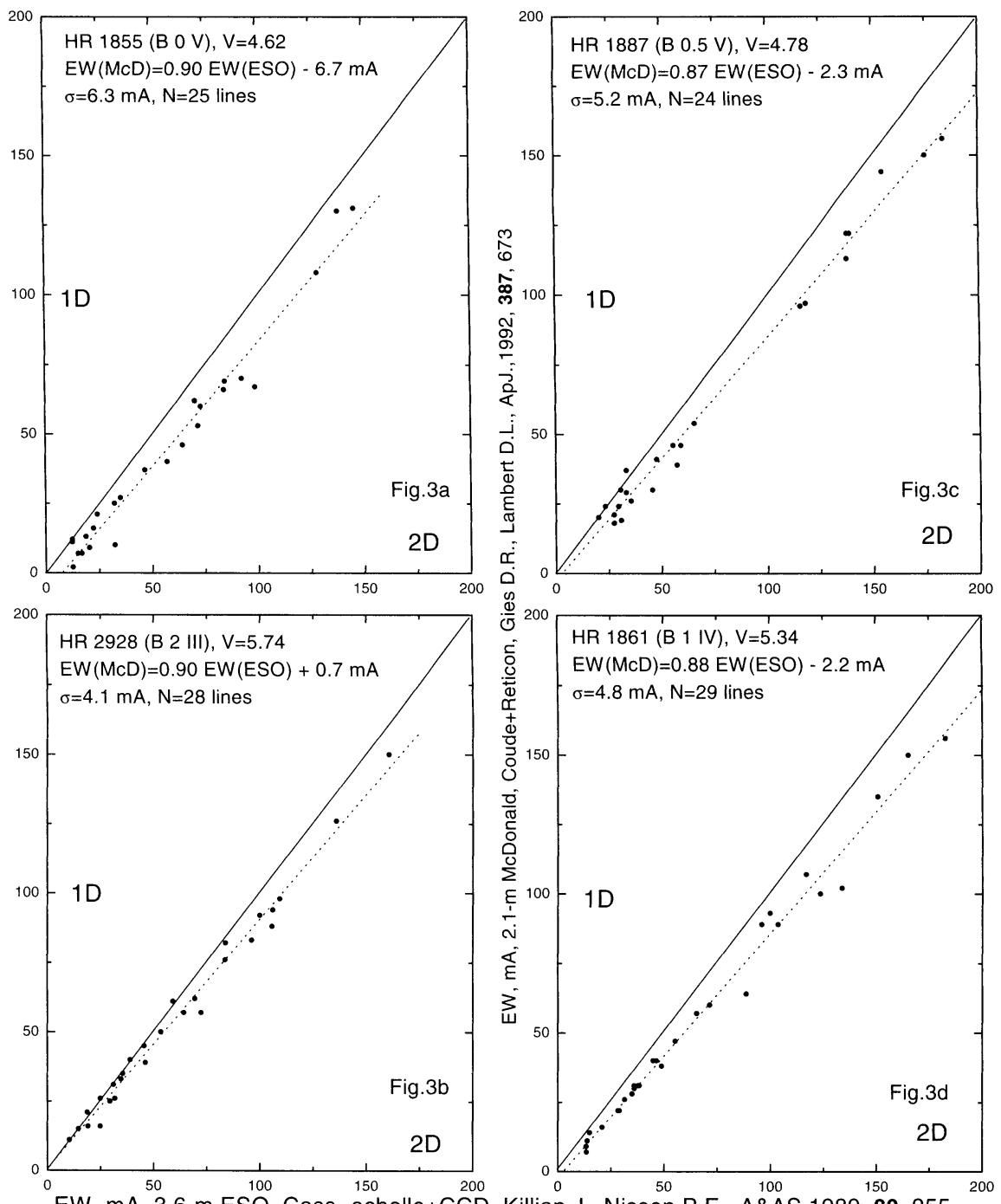

EW, mA, 3.6-m ESO, Cass.-echelle+CCD, Killian J., Nissen P.E., A\&AS, 1989, 80, 255

Figure 3. Comparison of EWs measured by 'classical' and echelle spectrographs for B stars 
used successfully for many decades in photometry). An extended version of this section is being prepared for publication.

\section{The Reduction of High Dispersion, High Signal-to-noise CCD Spectra}

The various steps in the reduction of CCD spectra are outlined below with emphasis on the processes that achieve high signal-to-noise $(\mathrm{S} / \mathrm{N})$. A software package dedicated solely to spectroscopic CCD reductions called CCDSPEC written by Austin Gulliver and Graham Hill incorporates these processes.

\subsection{Bias and Flat Field Frames}

Large numbers of bias and flat field frames are collected for each night of observing. These frames are averaged by applying a type of median filtering. A double pass, time-based median filtering of the bias and flat field data cubes has proven successful for unstable CCD pixels, an often ignored but common occurence. A histogram analysis checks for a skewed distribution and, if present, the median is determined solely for the most populous bin.

\subsection{Point Spread Function and Optimal Extraction}

The cross dispersion profile is determined using the master flat field or a stellar exposure. A digitized profile is constructed and fitted to each column of the spectrum along the dispersion. A high order polynomial is fitted to these measurements. If the spectrum is skewed with respect to the CCD, this is used to straighten the spectrum.

The fitting of the point spread function to each column of the frame is used to apply Horne's (1986) optimal extraction algorithm in which each pixel in a column is weighted as the inverse square of the noise. This optimal extraction achieves a $50 \%$ improvement over simple addition of the pixels in a given column.

\subsection{Scattered Light Removal}

Attention to this often neglected step in spectroscopic reductions has revealed its fundamental importance. The mean intensity of the scattered light is calculated above and below the stellar spectrum along the dispersion. On average the scattered light is $2.2 \%$ of the stellar intensity for the spectrographs investigated but its behaviour is unpredictable. It is generally variable along the dispersion, from star to star on a given night, sometimes from night to night for the same settings of a spectrograph and from one spectrograph to another. One spectrograph always shows a component of scattered light that includes the stellar spectrum at about $25 \%$ of the total scattered light. Patterns in the scattered light can persist during a night but not usually from night to night. A typical scattered light intensity is shown in Figure 4. The scattered light is removed by subtracting the smoothed, interpolated scattered light intensity from the stellar intensity. 


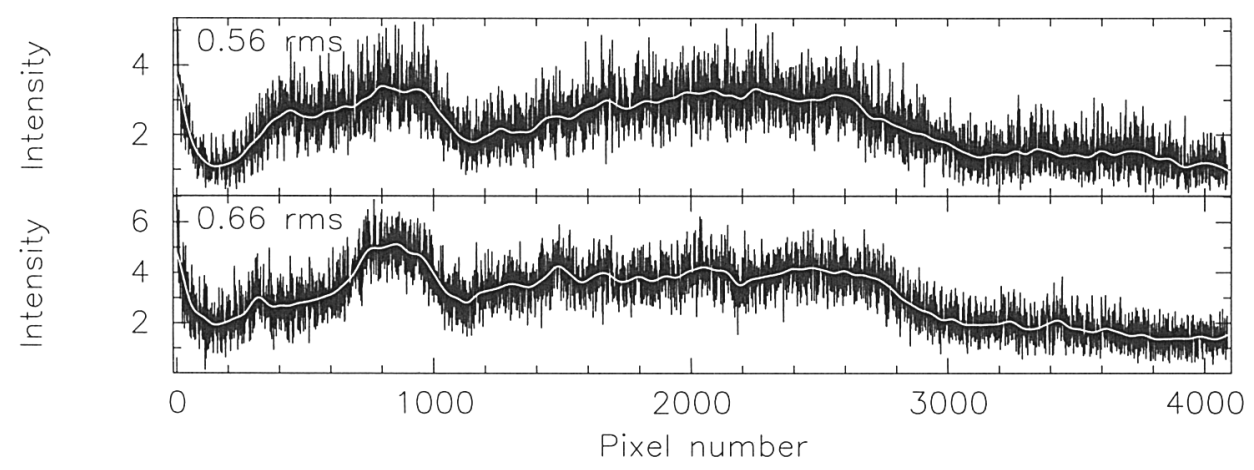

Figure 4. Fitted scattered light profiles above and below the spectrum.

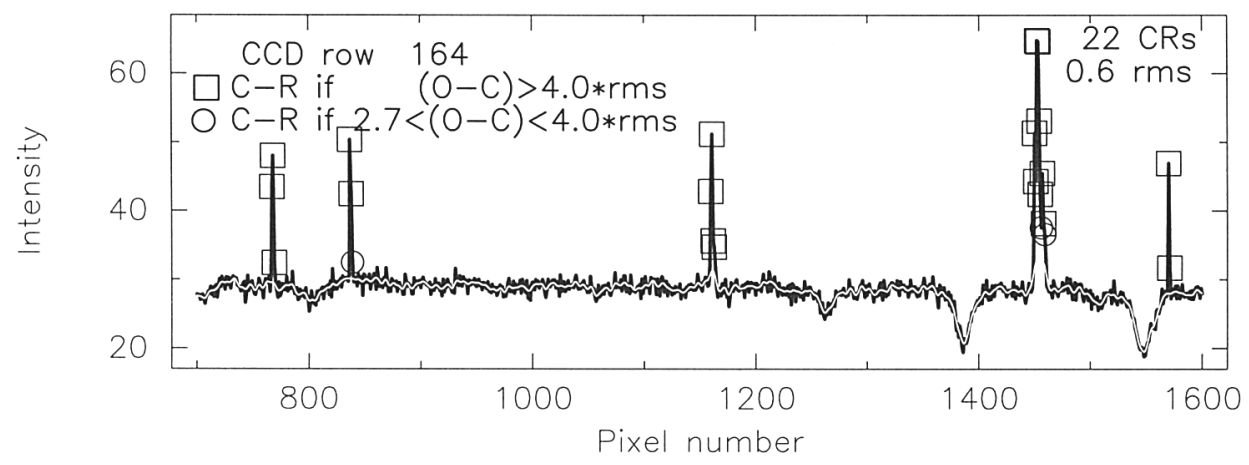

Figure 5. The removal of cosmic ray pixels using a FT fit to the stellar spectrum.

\subsection{Cosmic Ray Removal}

The effective removal of all cosmic ray artifacts from each exposure, whether short or long, is an issue that is usually accomplished by the comparison of multiple, successive short exposures. In a quest for the maximum $\mathrm{S} / \mathrm{N}$ possible we choose to remove all cosmic ray contaminated pixels from individual, long exposure CCD frames, a more difficult task. To do so, a Fourier Transform, with an appropriate filter window applied, is fitted to the stellar spectrum (see Figure 5).

This fit can be either parallel to the dispersion or perpendicular, or both. The filter window is chosen to match the resolution limit of the spectrograph. In most cases, cosmic rays are narrower than this limit and will not be fitted well. An rms deviation is calculated for the fit versus the spectrum and a rejection criterion for individual pixels is set at a user-selected multiple. Cosmic ray pixels are rejected if above this limit as are so-called 'skirt' pixels if they are contiguous and above a lower multiple.

The success rate of this automatic rejection is very high, in excess of $95 \%$. Figure 6 and Figure 7 show a 4800 second exposure of $\phi$ Cas reduced to one dimension. In Figure 6, there are 1922 cosmic ray pixels; 1898 (99\%) were found 


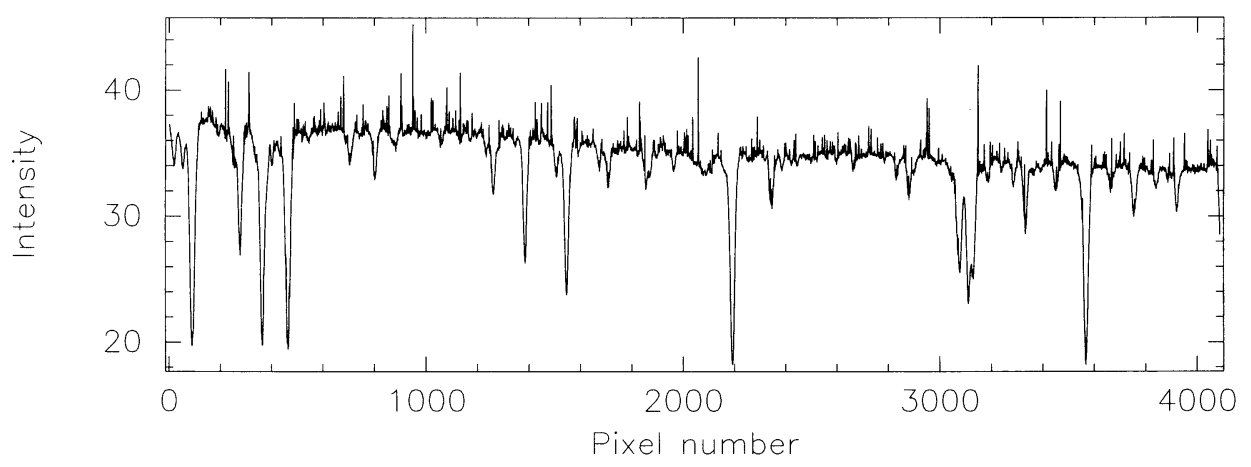

Figure 6. The reduced spectrum of $\phi$ Cas without cosmic ray removal.

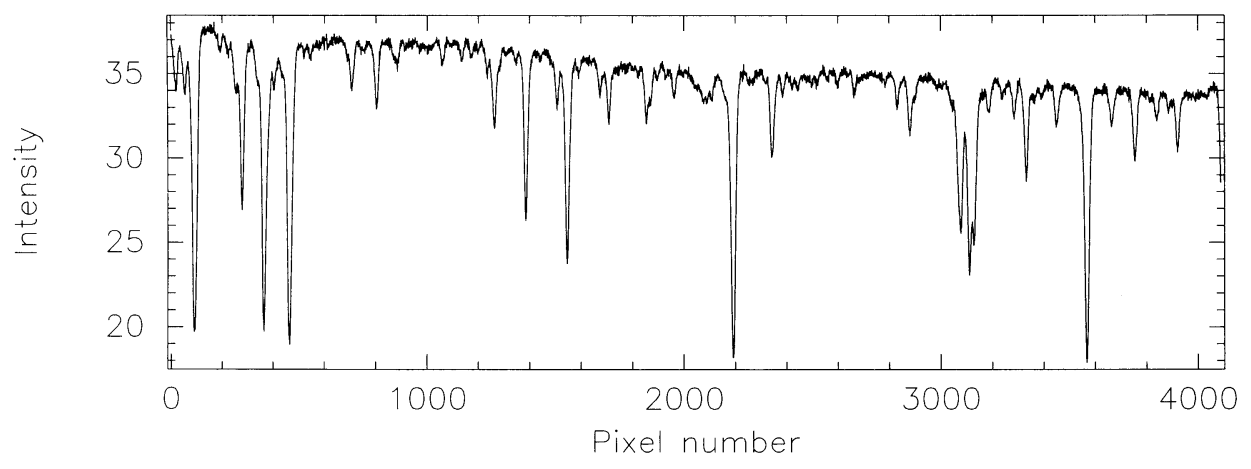

Figure 7. The clean spectrum of $\phi$ Cas with cosmic ray removal.

by the program. The remaining 24 were removed in a manual check. The final clean spectrum is shown in Figure 7. Because there can be unusual artifacts remaining, a visual inspection should always be performed. These artifacts are generally broad enough that the FT is able to fit them and rejection fails.

\subsection{Conclusion}

The results that can be attained using the process outlined above can be assessed by accessing www.brandonu.ca/physics/gulliver/ccd_atlases.html where some high dispersion, high $\mathrm{S} / \mathrm{N}$ spectral atlases can be displayed and manipulated. At this writing only Deneb is available but o Pegasi and Vega will soon follow.

\section{Atmospheric Extinction}

Extinction by the Earth's atmosphere generally follows the 'Bouguer Law': $m_{\lambda 0}=m_{\lambda}-k_{\lambda} X$, where $m_{\lambda 0}$ is stellar magnitude at a given wavelength $(\lambda)$ above Earth's atmosphere; $m_{\lambda}$ is observed stellar magnitude; $k_{\lambda}$ is extinction coefficient (mag./airmass); $X$ is the airmass relative to that at the zenith. 


\subsection{Airmass}

In the classical text, Hardie (1962) gives a polynomial formula for calculating airmass $(X)$ from zenith distance $(z)$. In his review, Young (1974) discussed airmass calculations and advocated the use of a simpler formula, $X=$ $\sec z\left[1-0.0012\left(\sec ^{2} z-1\right)\right]$, which is valid up to $\sec z=4$. Young also warns that airmasses greater than 4 should be avoided because of large random and systematic errors. If you really must observe closer to the horizon, the work of Kasten \& Young (1989) and Young (1994) should be consulted.

\subsection{Sources of Extinction}

Atmospheric extinction is comprised of four principal components (see Figure 8).

Rayleigh scattering Rayleigh scattering is proportional to $\lambda^{-4}$, with detailed formulae given by Allen (1973), Hayes \& Latham (1975) and Fröhlich \& Shaw (1980). Vertical extinction is proportional to local atmospheric pressure.

Aerosol scattering Aerosol scattering is due to dust particles, salt particles, water droplets, man-made pollutants in the earth's atmosphere (Hayes \& Latham 1975, Bruki et al. 1995, Forbes et al. 1996). We can model aerosol absorption using Angström's simple empirical approximation formula $A_{0} \lambda^{-\alpha}$. Aerosol extinction is quite variable, with $A_{0}$ showing diurnal and seasonal variations to a factor of two or more, and $0.5 \lesssim \alpha \lesssim 1.5$.

Ozone absorption Two bands to consider: the Huggins in the ultraviolet and the Chappuis in the optical, with two strong diffuse peaks around $5730 \AA$ and $6020 \AA$. High-resolution absorption coefficients are available (Burrows et al. 1999, Voigt et al. 2001). Ozone is concentrated at altitudes between 10 and $35 \mathrm{~km}$, and can vary significantly in a few hours (Hayes \& Latham 1975).

Telluric lines Several bands of discrete absorption lines, especially in the red and near-IR: oxygen $\left(\mathrm{O}_{2}\right)$ around $7590 \AA$ and $6870 \AA$, and water vapour $\left(\mathrm{H}_{2} \mathrm{O}\right)$ bands near $7100 \AA, 8090 \AA, 8920 \AA$, and $9277 \AA$. Water vapour is highly variable with irregular night-to-night and seasonal variations. The $\mathrm{H}_{2} \mathrm{O}$ bands do not follow the normal exponential absorption (Bouguer) law, but show a curve-ofgrowth effect (Hayes 1970).

\subsection{Variability of Extinction}

Atmospheric extinction is variable. This usually appears as a gradual decrease during the night, due to a slow fallout of aerosols (Young 1974). For a few hours, the extinction change can be represented by a linear function with time. Observationally, we must observe enough extinction stars to maintain a nearly continuous check on the extinction coefficients. Short exposures are required to avoid significant changes in airmass and extinction.

Young \& Irvine (1967) weighted each observation by $1 / \sec z$ so that residuals have units of mag./airmass. Thus residuals against time allows deviations from mean extinction to be spotted. 


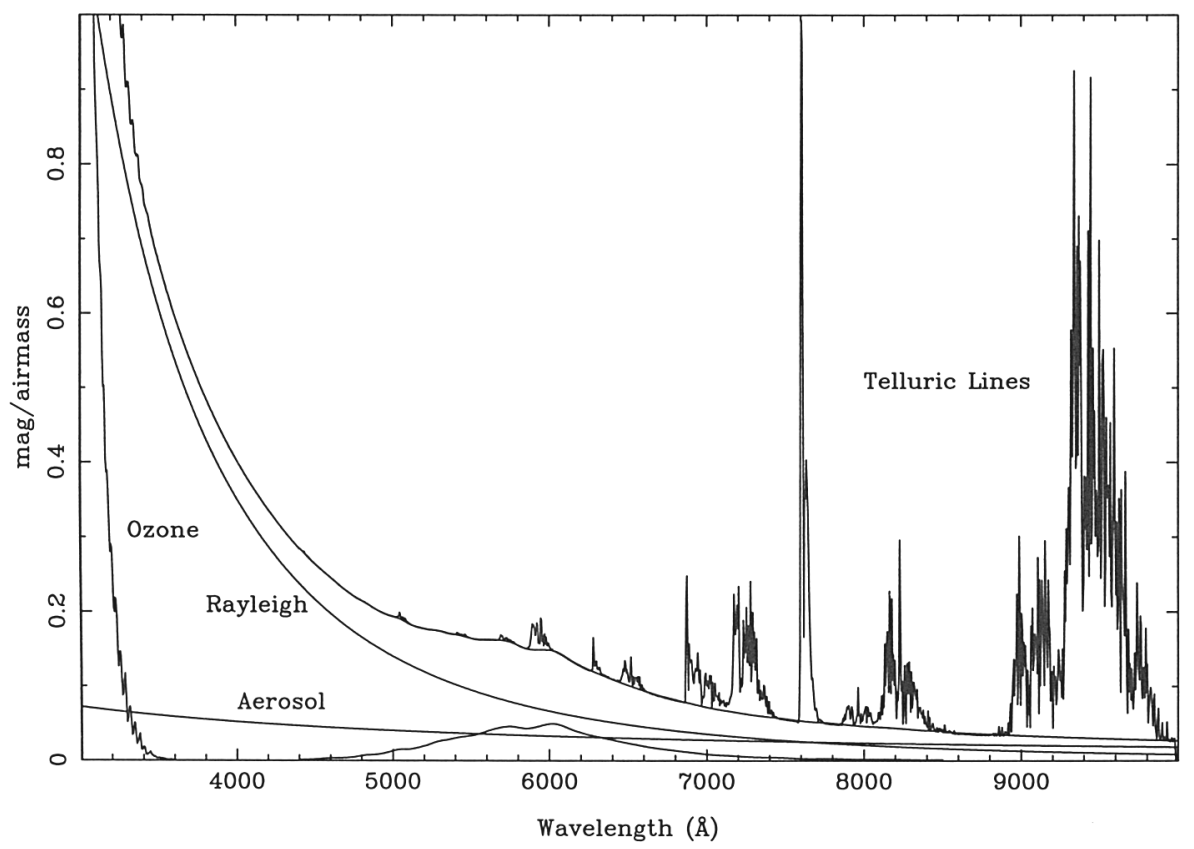

Figure 8. Simulation of extinction by the Earth's atmosphere, showing the relative contributions of the four sources mentioned in the text.

\subsection{Optimal Extinction Estimation}

Several papers discuss the reduction of photometry and attempt to produce an optimal reduction method (Harris et al. 1981; Popper 1982; SchwarzbergCzerny 1991; Manfroid \& Heck 1983, 1984). Similar methods could be applied to spectrophotometry, but the regions containing telluric lines will require special attention.

\subsection{Removal of Telluric Lines}

Traditional scanner spectrophotometry often just avoided telluric lines. This is undesirable and robust method of telluric line removal is required.

Honeycutt et al. (1977), McCord \& Clark (1979) and Cockran \& Barnes (1981) presented similar methods in which the telluric bands were treated as if they contained a linear term and a constant. The linear component was removed as per other wavelength regions, and the remaining part was treated as if it were part of the instrumental response.

Wade \& Horne (1988) used a non-linear model, $\left(\propto(\sec z)^{\alpha}\right)$. They found from observations between 1 and 2 airmasses, an average value of $\alpha=0.6$. Their method worked remarkably well, except in the cores of very strong bands.

The methods thus far considered cannot on their own give us an absolute value of telluric absorption. In order to do this we require a theoretical or 
empirical $\mathrm{H}_{2} \mathrm{O}$ transmission function that is tuned to the amount present at the time of observation.

Theoretical transmission functions can be calculated using the HITRAN molecular linelists (Rothman et al. 1998) and a model of the Earth's atmosphere (e.g. Nicholls 1988). Manduca \& Bell (1979) used IRTRANS (Traub \& Stier 1976) in their investigation of the atmospheric extinction in the near infrared. The model gave good agreement with observations. They concluded that no simple rule of thumb such as the 'square root law' is adequate for estimating the true extinction.

Galkin \& Arkharov (1981) presented an empirical method. They concluded that their methods allows for magnitude determinations within the telluric bands with an error of $3-5 \%$.

Overall some sort of modeling is required to correct for telluric lines, but even then we may not be able to obtain a perfect correction.

\subsection{Bandpass effects}

At the resolution of the ASTRA instrument (Adelman et al. 2002) the individual telluric lines are not resolved. Thus, flux through each resolution element will depend on the nature of the intrinsic telluric lines and any stellar features within that region. Strong absorption (or emission) features may be hidden by the lines or 'visible' between them. We cannot a priori know which case is occurring in each band (e.g. Stevenson 1994).

\section{Flux Standards}

Spectrophotometric observations must be placed on an absolute flux scale by reference to stars with known values of true flux at top of Earth's atmosphere. Standard stars have been calibrated against terrestrial sources of known properties. Unfortunately, very few stars have been calibrated directly. The primary standard is $\alpha$ Lyr (Vega). The standard references for absolute fluxes are Hayes \& Latham (1975), Tüg et al. (1977) and Hayes (1985). The accuracy of these an other calibrations was assessed by Mégessier (1995).

Tüg et al. (1977) also presented absolute fluxes for 109 Vir and Tüg (1980) obtained absolute flux calibrations for 14 southern stars.

Most calibrations are at relatively low resolution (typically $50-100 \AA$ ). Corrections for bandpass effects need to be considered, which may require the use of high-resolution spectra. Colina et al. (1996) presented a calibration for Vega, for use with HST data, which used Kurucz ATLAS9 fluxes to generate a higherresolution.

The available absolute calibrations are accurate to typically $1-2 \%$. Ultimately this uncertainty will limit the accuracy of the final fluxes of other stars. However, the internal precision can be significantly higher, and should moreaccurate absolute calibrations become available the fluxes can be re-calibrated to higher accuracy. 


\section{The ASTRA Spectrophotometer}

The ASTRA (Automatic Spectrophotometric Telescope Research Associates) Spectrophotometer is discussed by Adelman et al. (2002). It will be located at Washington Camp, AZ, the Fairborn Observatory site, at $1800 \mathrm{~m}$, a few kilometers east of Nogales, AZ. Its spectral resolution will be $7 \AA$ for $\lambda \lambda \leq 3000$ 6000 and $14 \AA$ for $\lambda \lambda 5500-\geq 9000$. To obtain $\mathrm{S} / \mathrm{N} \geq 100$ over this region will take approximately $15 \mathrm{~s}$ for an $\mathrm{A} 0 \mathrm{~V}, \mathrm{~V}=5.0$ star. The major uncertainties are the wavelength range and the number of read electrons per pixel. For bright stars exposure times will be about one minute to obtain higher $\mathrm{S} / \mathrm{N}$ data. The site is expected to have 130 to 180 photometric nights per year. The declination coverage will be approximately $+75^{\circ}$ to $-35^{\circ}$. Initially about 10 minutes each hour will be used to obtain calibration and extinction observations.

The first major projects are the revision and extension of the secondary standards and sample fluxes of Population I and II stars with the auxiliary projects synthetic colors and line indices. In addition large variety of observing projects for specific physical problems and types of stars are expected. As the data anticipated to be obtained by ASTRA each year exceeds the capability of three project astronomers, they are looking for research collaborators. Colleagues who desire to have local standards for comparison with selected variables should contact Adelman, Gulliver, and/or Smalley as soon as possible. The project's home page at http://www.citadel.edu/physics/astra/index.html will contain additional information and report developments as they occur.

Acknowledgments. We are grateful to the Scientific Organizing Committee for the time to present this round table. IB and his colleagues are grateful to RFBR (grants 02-02-17174 and 02-02-17423) for financial support.

\section{References}

Adelman, S. J., Gulliver, A. F., Smalley, B., Pazder, J. S., Younger, P. F., Boyd, L., \& Epand, D., 2002, IAU Symposium 210 Poster papers, p. TBD

Allen C.W., 1973, Astrophysical Quantities, Athlone Press, London, 3rd Edition. Burki G., Rufener F., Burnet M., Richard C., Blecha A., Bratschi P., 1995, A\&AS, 112, 383.

Burrows J.P., Dehn A., Deters B., Himmelmann S., Richter A., Voigt S., Orphal J., 1999, JQSRT, 61, 509.

Cochran A.L., Barnes T.G., 1981, ApJS, 45, 73.

Colina L., Bohlin R.C., Castelli F., 1996, Instrument Science Report, CAL/SCS008, (Baltimore: STScI).

Forbes M.C., Dodd R.J., Sullivan D.J., 1996, Baltic Astronomy, 5, 281.

Fröhlich C., Shaw G.E., 1980, Applied Optics, 19, 1773.

Galazutdinov, G.A., 1992, SAO RAS Preprint N 92

Galkin V.D., Arkharov A.A., 1981, Astron. Zh., 58, 636 [Sov. Astron., 25, 361]. Harris W.E., Fitzgerald M.P., Reed B.C., 1981, PASP, 93, 507.

Honeycutt R.K., Ramsey L.W., Warren W.H., Ridgway S.T., 1977, ApJ, 215, 584 . 
Hardie R.H., 1962, in Astronomical Techniques (Hittner W.A. ed.), Chap. 8, p. 178, Univ of Chicago Press.

Hayes D.S., 1970, ApJ, 159, 165.

Hayes D.S., Latham D.W., 1975, ApJ, 197, 593.

Hayes D.S., 1985, in Calibration of Fundamental Stellar Quantities (Hayes D.S., Pasinetti L.E., Philip A.G.D., eds.) IAU Symp. 111, p. 225.

Horne, K., 1986, PASP, 98, 609

Kasten F., Young A.T., 1989, Applied Optics, 28, 4735.

Manduca A., Bell R.A., 1979, PASP, 91, 848.

Manfroid J., Heck A., 1983, A\&A, 120, 302.

Manfroid J., Heck A., 1984, A\&A, 132, 110.

McCord T.B., Clark R.N., 1979, PASP, 91, 571.

Mégessier C., 1995, A\&A, 296, 771.

Musaev, F.A., Galazutdinov G.A., Sergeev, A.V., Karpov, N.V., Pod'yachev, Yu.V., 1999, Kinematics and physics of celestial bodies, 13, 282

Nicholls R.W., 1988, JQSRT, 40, 275.

Popper D.M., 1982, PASP, 94, 204.

Rothman L.S., et al., 1998, JQSRT, 60, 665.

Schwarzenberg-Czerny A., 1991, A\&A, 252, 425.

Stevenson C.C., 1994, MNRAS, 267, 904.

Traub W.A., Stier M.T., 1976, Applied Optics, 15, 364.

Tüg H., 1980, A\&A, 82, 195.

Tüg H., White N.M., Lockwood G.W., 1977, A\&A, 61, 679.

Voigt S., Orphal J., Bogumil K., Burrows J.P., 2001, J. Photochem. Photobiol. A, $143,1$.

Wade R.A., Horne K., 1988, ApJ, 324, 411.

Young A.T., 1974, Methods of Experimental Physics 12A (New York Academic Press), Chap. 3, p. 123.

Young A.T., 1994, Applied Optics, 33, 1108.

Young A.T, Irvine W.M., 1967, AJ, 72, 945. 